\title{
Murine Fig4 is dispensable for muscle development but required for muscle function
}

\author{
Aaron Reifler ${ }^{1,5}$, Guy M Lenk², Xingli Li ${ }^{1}$, Linda Groom³ ${ }^{3}$ Susan V Brooks ${ }^{4}$, Desmond Wilson ${ }^{1}$, Michyla Bowerson ${ }^{1}$, \\ Robert T Dirksen ${ }^{3}$, Miriam H Meisler ${ }^{2}$ and James J Dowling ${ }^{1 *}$
}

\begin{abstract}
Background: Phosphatidylinositol phosphates (PIPs) are low-abundance phospholipids that participate in a range of cellular processes, including cell migration and membrane traffic. PIP levels and subcellular distribution are regulated by a series of lipid kinases and phosphatases. In skeletal muscle, PIPs and their enzymatic regulators serve critically important functions exemplified by mutations of the PIP phosphatase MTM1 in myotubular myopathy (MTM), a severe muscle disease characterized by impaired muscle structure and abnormal excitation-contraction coupling. FIG4 functions as a PIP phosphatase that participates in both the synthesis and breakdown of phosphatidylinositol 3,5-bisphosphate $\left(\mathrm{PI}(3,5) \mathrm{P}_{2}\right)$. Mutation of $F / G 4$ results in a severe neurodegenerative disorder in mice and a progressive peripheral polyneuropathy in humans. The effect of FIG4 mutation on skeletal muscle has yet to be examined.
\end{abstract}

Methods: Herein we characterize the impact of FIG4 on skeletal muscle development and function using the spontaneously occurring mouse mutant pale tremor $(p / t)$, a mouse line with a loss of function mutation in Fig4.

Results: In plt mice, we characterized abnormalities in skeletal muscle, including reduced muscle size and specific force generation. We also uncovered ultrastructural abnormalities and increased programmed cell death. Conversely, we detected no structural or functional abnormalities to suggest impairment of excitation-contraction coupling, a process previously shown to be influenced by $\mathrm{PI}(3,5) \mathrm{P}_{2}$ levels. Conditional rescue of Fig4 mutation in neurons prevented overt muscle weakness and the development of obvious muscle abnormalities, suggesting that the changes observed in the plt mice were primarily related to denervation of skeletal muscle. On the basis of the ability of reduced FIG4 levels to rescue aspects of Mtmr2-dependent neuropathy, we evaluated the effect of Fig4 haploinsufficiency on the myopathy of Mtm1-knockout mice. Male mice with a compound Fig $4^{+/-} / \mathrm{Mtm}^{1-N}$ genotype displayed no improvements in muscle histology, muscle size or overall survival, indicating that FIG4 reduction does not ameliorate the Mtm1-knockout phenotype.

Conclusions: Overall, these data indicate that loss of Fig4 impairs skeletal muscle function but does not significantly affect its structural development.

Keywords: Autophagy, Congenital myopathies, FIG4, MTM1, Phosphatidylinositol

\section{Background}

Phosphatidylinositol phosphates (PIPs) are low-abundance phospholipids that are implicated as regulators of a range of cellular processes, including cell migration, subcellular organelle trafficking and autophagy [1,2]. Phosphatidylinositol 3,5-bisphosphate, or $\mathrm{PI}(3,5) \mathrm{P}_{2}$, is a low-abundance PIP whose function has recently come into

\footnotetext{
* Correspondence: jamedowl@umich.edu

'Department of Pediatrics, University of Michigan Medical Center, Ann Arbor, MI 48109-2200, USA

Full list of author information is available at the end of the article
}

focus [3]. $\mathrm{PI}(3,5) \mathrm{P}_{2}$ is generated by the action of PIKfyve, a five-position phosphoinositide kinase [4]. $\mathrm{PI}(3,5) \mathrm{P}_{2}$ is metabolized by FIG4 (Sac3), a five-position phosphoinositide phosphatase, to regenerate phosphatidylinositol 3phosphate $(\mathrm{PI}(3) \mathrm{P})$, and by myotubularins, a family of three-position phosphatases to generate phosphatidylinositol 5-phosphate (or PI(5)P) [5-8]. Of note, maximal function of PIKfyve requires a complex of proteins that includes VAC14 and FIG4 [9-11]. Reduced abundance of FIG4 protein destabilizes PIKfyve, resulting in a threefold reduction of $\mathrm{PI}(3,5) \mathrm{P}_{2}$ relative to total PI levels [12].

\section{Biomed Central}


We recently identified and characterized a spontaneous mouse mutant ("pale tremor" or plt) with a homozygous recessive loss-of-function mutation in the Fig4 gene [12]. plt mice exhibit severe and progressive neurodegeneration that involves both neurons and glia of the central and peripheral nervous system [13]. The main subcellular phenotype is increased vacuolization due to defective lysosomal function and impaired autophagy $[14,15]$. In addition, we (the Meisler group) have identified recessive FIG4 mutations in patients with hereditary peripheral neuropathy (Charcot-Marie-Tooth Disease type 4J) [16-18] and motor neuron disease (amyotrophic lateral sclerosis, or ALS) [19]. However, the role of FIG4 in skeletal muscle has yet to be extensively examined in detail.

Studies of myotubularins indicate a potentially important role for $\mathrm{PI}(3,5) \mathrm{P}_{2}$ regulation in skeletal muscle [20-22]. The mammalian myotubularin gene family contains 15 members encoding phosphatases that primarily dephosphorylate $\mathrm{PI}(3) \mathrm{P}$ and $\mathrm{PI}(3,5) \mathrm{P}_{2}$ [23]. Mutation of MTM1, the canonical member of this gene family, results in increased levels of $\mathrm{PI}(3) \mathrm{P}$, and possibly $\mathrm{PI}(3,5) \mathrm{P}_{2}$, in skeletal muscle and causes myotubular myopathy (MTM), a severe congenital muscle disease associated with altered muscle structure and profound muscle weakness [24]. Mutation of MTM1 compromises multiple aspects of muscle function [25], most notably excitation-contraction coupling (EC coupling) [26], the process by which neuronal stimulation to muscle is translated into calcium-dependent muscle contraction. Specifically, MTM1 mutations lead to severe abnormalities in the structure of the EC coupling machinery [27].

MTMR14 is another myotubularin family member that is important for muscle function [26]. Loss of MTMR14 function in zebrafish and mice causes aberrant autophagy and impaired EC coupling [26,28]. Unlike MTM1, loss of MTMR14 alters EC coupling without significantly changing the underlying structure of the EC coupling machinery. Direct application of $\mathrm{PI}(3,5) \mathrm{P}_{2}$ increases calcium release from microsomes containing the intracellular ryanodine receptor 1 calcium release channel (RyR1), a critical component of the EC coupling apparatus. Thus, direct regulation of RyR1-dependent stimulated calcium release may represent one important role of $\mathrm{PI}(3,5) \mathrm{P}_{2}$ and MTMR14 [29].

Given the potential importance of $\mathrm{PI}(3,5) \mathrm{P}_{2}$ in skeletal muscle, we sought to understand the impact of FIG4 mutation on muscle development and homeostasis. To address this issue, we examined skeletal muscle structure and function in the plt-null mouse model of FIG4 dysfunction. We examined plt mice, which die before 2 months of age as a result of progressive neurodegeneration, as well as plt mice with restored FIG4 expression in neurons $[12,15]$. The latter mice have no overt phenotype and survive for more than 18 months. Our data reveal that Fig4 mutation is associated with skeletal muscle changes (atrophy and increased apoptosis) and impaired muscle force generation, but not with abnormalities in the structure or function of the EC coupling machinery. The changes are likely the consequence of impaired neuronal input because phenotypic rescue is largely provided by neuronal expression of FIG4. In addition, we found that haploinsufficiency of Fig4 does not ameliorate effects of Mtm1 mutation in muscle, in contrast to a previous report that haploinsufficiency of Fig4 rescues the neuropathy associated with Mtmr2 mutation [30,31]. Together, our results support a requirement for FIG4 in skeletal muscle function, but not a cellular autonomous role in either muscle development or EC coupling.

\section{Methods}

\section{Animal care and husbandry}

All animals were cared for per protocol under the guidance of, and with ethical approval from, the University Committee on Use and Care of Animals (UCUCA) and with the assistance of members of the University of Michigan's Unit for Laboratory Animal Medicine (ULAM), who carefully monitored the health of the rodent colonies. ULAM maintained proper environmental regulation, including temperature and light cycles, unlimited access to water, appropriate food supply and clean enclosures. The Fig4-null mutation plt is maintained on two congenic lines, C57BL/6J.plt/+ (N14) and C3H.plt/+ (N10) [32]. Experiments were carried out on homozygous plt/plt F1 mice obtained from crosses between the two congenic strains. Pups were weaned according to standard protocols, and tails were clipped for genotyping.

\section{Western blot analysis}

Western blot analyses were performed using the following antibodies: FIG4 (1:1,000 NeuroMab; UC Davis/NIH NeuroMab Facility, Davis, CA, USA) and glyceraldehyde 3-phosphate dehydrogenase (1:1,000 GAPDH; Millipore, Billerica, MA, USA). Mouse multitissue Western blot antibody was obtained from IMGENEX (San Diego, CA, USA). Protein extracts were established from flashfrozen mouse skeletal muscle and brain using T-PER tissue protein extraction reagent and a Dounce tissue homogenizer (Pierce Biotechnology, Rockford, IL, USA). Approximately $50 \mu \mathrm{g}$ of protein were loaded per sample, resolved by polyacrylamide gel electrophoresis on $11 \%$ gels, and transferred to polyvinylidene fluoride. Secondary antibodies were used at 1:2,000 (Santa Cruz Biotechnology, Santa Cruz, CA, USA), blots were developed using electrochemiluminescence reagent (GE Biosciences, Pittsburgh, PA, USA), and bands visualized using the Bio-Rad ChemiDoc XRS + System illuminator (Bio-Rad Laboratories, Hercules, CA, USA). 


\section{Histopathology}

Animals were killed by anesthetic injection, which was followed by cervical dislocation. Tissues were then isolated using sterile surgical methods without the use of laminar flow hoods. Muscle tissue from quadriceps and tibialis anterior muscles was dissected and mounted onto small balsa wood pieces that had previously been frozen with drops of Tissue-Tek O.C.T. compound (Sakura Finetek USA, Torrance, CA, USA) and then semithawed with light friction. The mounted muscle tissue was immediately submerged in a $-55^{\circ} \mathrm{C}$ isopentane bath cooled by liquid nitrogen for flash-freezing.

Muscles were cut into $12-\mu \mathrm{m}$ cross-sections and mounted on Superfrost Plus slides (Thermo Scientific, Waltham, MA, USA) using a Leica cryostat (Leica Biosystems, Buffalo Grove, IL, USA) at $-20^{\circ} \mathrm{C}$ and dried at room temperature before storage at $-80^{\circ} \mathrm{C}$. Slides were stained with Mayer's hematoxylin and eosin (H\&E) or succinate dehydrogenase (SDH) following standard protocols and mounted with Permount mounting medium (Thermo Scientific). Apoptotic fibers were visualized with the ApopTag Plus Peroxidase In Situ Apoptosis Kit (S7101; Chemicon International/EMD Millipore, Billerica, MA, USA). Photomicrographs were captured using an INFINITY1 digital camera with eponymous software (Lumenera Corp, Ottawa, ON, Canada) visualized through an Olympus BX43 light microscope (Olympus America, Center Valley, PA, USA).

\section{Ultrastructural analysis}

Immediately following dissection, quadriceps and gastrocnemius muscles were carefully cut into approximately $1-\mathrm{mm} \times 2-\mathrm{mm}$ fragments and incubated in Karnovsky's fixative overnight at $4^{\circ} \mathrm{C}$. Fixed tissue was brought to the Microscopy and Imaging Laboratory (MIL) Core facility at the University of Michigan for processing. Ultrathin sections were analyzed for orientation, and grids were prepared for use on the Philips CM-100 transmission electron microscope (Koninklijke Philips N.V., Amsterdam, The Netherlands).

\section{Myocyte isolation}

Muscle was dissected from the shoulders and legs of dead mice and placed immediately into sterile phosphatebuffered saline. Muscle was then minced finely with a sterile razor, fully dissociated with a mixture of collagenase type I $(0.1 \%)$ and trypsin $(0.1 \%)$ in Ham's F-12 medium, then incubated at $37^{\circ} \mathrm{C}$ for approximately $1 \mathrm{~h}$ with periodic trituration. Cells were pelleted and resuspended in 1:1 Dulbecco's Modified Eagle Medium:Ham's F-12 Nutrient Mixture (DMEM/F-12) (Gibco/Life Technologies, Grand Island, NY, USA) with $20 \%$ fetal calf serum (HyClone Laboratories, Logan, UT, USA), then filtered through 70 - and 40- $\mu \mathrm{m}$ meshes and plated onto collagen-coated dishes (BD Biosciences, San Jose, CA, USA). Media were changed after a 1 -h incubation at $37^{\circ} \mathrm{C}$, and recombinant human fibroblast growth factor-basic (AA 10-155, Publication PHG0026; Gibco/Life Technologies) was added to a final concentration of $10 \mathrm{ng} / \mathrm{ml}$. Cells were maintained at $37^{\circ} \mathrm{C}$ in a $5 \% \mathrm{CO}_{2}$ atmosphere with daily changes of fresh media. Cells were visualized using Hamamatsu ORCA-R2 camera and software (Hamamatsu Photonics, Hamamatsu-shi, Japan) on a Leica inverted microscope (Leica Microsystems). All experimentwas were performed on passages 2 through 4 myocytes. Terminal deoxynucleotidyl transferase-mediated dUTP nick end labeling (TUNEL) staining was performed per the manufacturer's recommendations on myocytes grown on coverglass coated with fibronectin and fixed with $4 \%$ paraformaldehyde prior to staining.

\section{Muscle force measurement}

Extensor digitorum longus (EDL) and soleus muscles were carefully isolated and removed from anesthetized mice. Muscles were immediately placed into a bath of Krebs mammalian Ringer solution with $0.25 \mathrm{mM}$ tubocurarine chloride maintained at $25^{\circ} \mathrm{C}$ and bubbled with $95 \% \mathrm{O}_{2}$ and $5 \% \mathrm{CO}_{2}$ to stabilize $\mathrm{pH}$ at 7.4. Using 5-0 silk suture, the distal tendon of the muscle was attached to a servomotor (model 305B; Aurora Scientific, Aurora, ON, Canada), and the proximal tendon was attached to a force transducer (model BG-50; Kulite Semiconductor Products, Leonia, NJ, USA). Muscles were stimulated by square pulses delivered by two platinum electrodes connected to a high-power biphasic current stimulator (model 701B; Aurora Scientific). A personal computer running custom-designed software (LabVIEW 7.1; National Instruments, Austin, TX, USA) controlled electrical pulse properties and servomotor activity and recorded data from the force transducer. Stimulation voltage and optimal muscle length $\left(L_{\mathrm{o}}\right)$ were adjusted to give maximum twitch force [33]. While held at $L_{\mathrm{o}}$, muscles were subjected to trains of pulses $300 \mathrm{~ms}$ in duration for EDL muscles and $900 \mathrm{~ms}$ for soleus muscles, with increasing stimulation frequency until maximum isometric tetanic force $\left(P_{o}\right)$ was achieved [33]. $L_{\mathrm{o}}$ was measured with digital calipers, and muscle fiber lengths $\left(L_{\mathrm{f}}\right)$ were determined by multiplying $L_{\mathrm{o}}$ by previously established $L_{\mathrm{f}}$-to- $L_{\mathrm{o}}$ ratios of 0.44 for EDL muscle and 0.71 for soleus muscle [33]. Total muscle fiber cross-sectional area (CSA) was estimated by dividing the mass of the muscle by the product of $L_{\mathrm{f}}$ and $1.06 \mathrm{~g} / \mathrm{cm}^{3}$, the density of mammalian skeletal muscle. $P_{o}$ was normalized by CSA to give specific $P_{o}$.

\section{Simultaneous measurement of macroscopic L-type $\mathrm{Ca}^{2+}$ currents and voltage-gated $\mathrm{Ca}^{2+}$ transients in myotubes} Primary cultures of skeletal myotubes were generated from myoblasts derived from wild-type (WT) and plt mice as previously described [34]. The whole-cell voltage-clamp 
technique in conjunction with a $\mathrm{Ca}^{2+}$-sensitive dye (fluo-4) was used to simultaneously measure voltage-gated L-type $\mathrm{Ca}^{2+}$ currents (L-currents) and intracellular $\mathrm{Ca}^{2+}$ transients on individual myotubes from 8- to 11-day-old myotube cultures [34,35]. All voltage-clamp experiments were carried out after an approximately 5-min period of dialysis following establishment of the whole-cell configuration. The external recording solution consisted of $145 \mathrm{mM}$ tetraethylammonium chloride, $10 \mathrm{mM} \mathrm{CaCl}_{2}$ and $10 \mathrm{mM}$ 2-[4-(2-hydroxyethyl)piperazine-1-yl]ethanesulfonic acid (HEPES) ( $\mathrm{pH}$ 7.4). The internal patch pipette solution consisted of $145 \mathrm{mM}$ Cs-aspartate, $10 \mathrm{mM} \mathrm{CsCl}, 0.1 \mathrm{mM}$ $\mathrm{Cs}_{2}$-ethylene glycol tetraacetic acid, $1.2 \mathrm{mM} \mathrm{MgCl} 2,5 \mathrm{mM}$ Mg-ATP, $0.2 \mathrm{mM} \mathrm{K}_{5}$-fluo-4 and $10 \mathrm{mM}$ HEPES (pH 7.4). A 1-s prepulse to $-30 \mathrm{mV}$ delivered immediately before each test pulse was used to inactivate voltage-gated $\mathrm{Na}^{+}$and T-type $\mathrm{Ca}^{2+}$ channels without producing significant L-channel inactivation. L-currents and $\mathrm{Ca}^{2+}$ release were subsequently elicited by $200-\mathrm{ms}$ test depolarizations from $-50 \mathrm{mV}$ to $+70 \mathrm{mV}$ in $10-\mathrm{mV}$ increments and a $10-\mathrm{s}$ interval between each test pulse. Capacitative currents were minimized to about $10 \%$ using the capacitance cancelation feature of the patch-clamp amplifier. Remaining linear components were leak-subtracted using a $\mathrm{P} / 3$ protocol delivered from a holding potential of $-80 \mathrm{mV}$ before each test pulse. Peak L-current magnitude was normalized to cell capacitance $(\mathrm{pA} / \mathrm{pF})$, which was plotted as a function of membrane potential $\left(V_{\mathrm{m}}\right)$ and fitted as $I=\mathrm{G}_{\max }$ $\left(V_{\mathrm{m}}-V_{\text {rev }}\right) /\left(1+\exp \left[\left(V_{\mathrm{G} 1 / 2}-V_{\mathrm{m}}\right) / k_{\mathrm{G}}\right]\right)$, where $G_{\mathrm{max}}$ is the maximal L-channel conductance, $V_{\mathrm{m}}$ is test potential, $V_{\mathrm{rev}}$ is extrapolated reversal potential, $V_{\mathrm{G} 1 / 2}$ is the voltage for half-maximal activation of $G_{\max }$, and $k_{\mathrm{G}}$ is a slope factor. Relative changes in intracellular $\mathrm{Ca}^{2+}$ during each test depolarization were measured following dialysis with $\mathrm{K}_{5^{-}}$ fluo-4 salt. Fluo-4-dialyzed myotubes were excited at 480 $\mathrm{nm}$ and fluorescence emission measured at $535 \mathrm{~nm}$ was digitized at $10 \mathrm{kHz}$. A computer-controlled shutter was used to eliminate dye illumination during intervals between each test pulse. Relative peak changes in intracellular $\mathrm{Ca}^{2+}$ were expressed as $\Delta \mathrm{F} / \mathrm{F}\left(\left[\mathrm{F}_{\text {peak }}-\mathrm{F}_{\text {base }}\right] / \mathrm{F}_{\text {base }}\right)$ at the end of each test pulse, plotted as a function of $V_{\mathrm{m}}$, and fitted according to the equation $\Delta \mathrm{F} / \mathrm{F}=\left(\Delta \mathrm{F} / \mathrm{F}_{\max }\right) /\{1+\exp$ $\left.\left[\left(V_{\mathrm{F} 1 / 2}-V_{\mathrm{m}}\right) / k_{\mathrm{F}}\right]\right\}$, where $(\Delta \mathrm{F} / \mathrm{F})_{\max }$ is the calculated maximal change in fluorescence, $V_{\mathrm{F} 1 / 2}$ is the voltage for half-maximal activation of $(\Delta \mathrm{F} / \mathrm{F})_{\max }$, and $k_{\mathrm{F}}$ is a slope factor. Pooled current-voltage $(I-V)$ and fluorescencevoltage $(\Delta \mathrm{F} / \mathrm{F}-\mathrm{V})$ data were expressed as means $\pm \mathrm{SEM}$. Statistical significance was determined using a two-tailed Student's $t$-test.

\section{Statistical analyses}

GraphPad Prism software (GraphPad Software, La Jolla, CA, USA) was used to calculate the significance of fiber size differences and TUNEL staining results with unpaired
Student's $t$-tests and one-way analysis of variance with Tukey's post hoc multiple comparison test.

\section{Results}

\section{FIG4 is expressed in skeletal muscle}

We wanted to establish that FIG4 protein was present in skeletal muscle. We therefore performed Western blot analysis using anti-FIG4 antibody (NeuroMab). We first used a premade multitissue Western blot antibody (IMGENEX) and found that FIG4 was present in most tissues examined, including skeletal muscle (Figure 1A). To determine if FIG4 expression was regulated with maturation of skeletal muscle, we examined protein extracts from skeletal muscle of various postnatal ages (Figure 1B) as well as from differentiating $\mathrm{C} 2 \mathrm{C} 12$ myocytes (Figure $1 \mathrm{C}$ ). We detected no obvious variability in FIG4 levels at

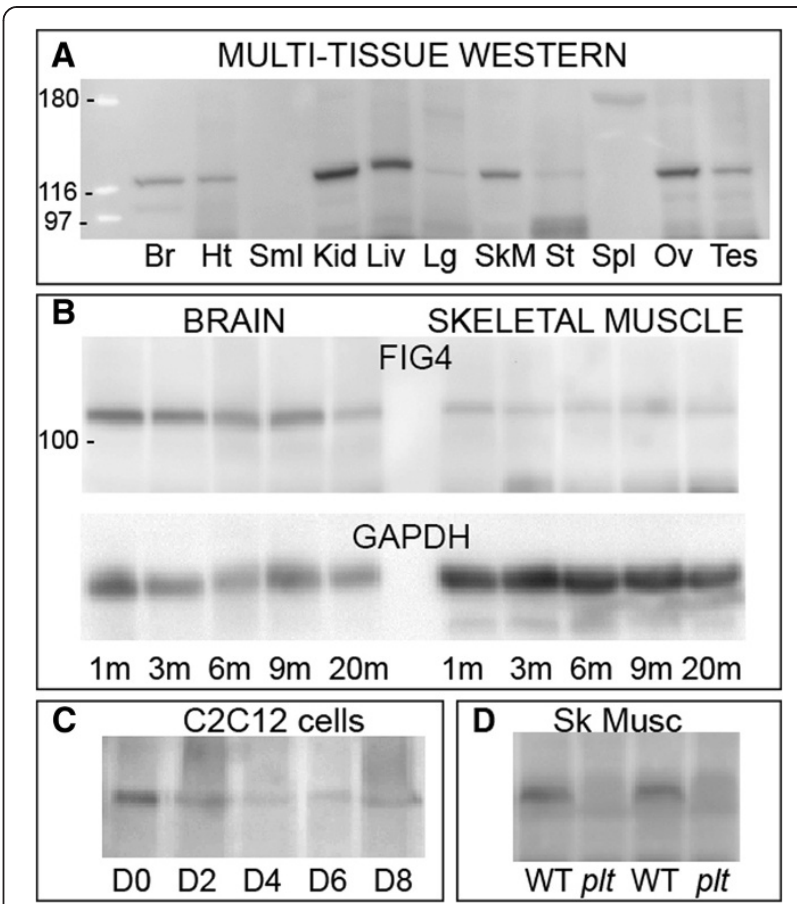

Figure 1 FIG4 is expressed in skeletal muscle. Western blot analysis was performed to establish FIG4 expression using anti-FIG4 antibody. (A) Mouse multitissue Western blot reveals expression of FIG4 in a variety of tissues $(\mathrm{Br}=$ brain, $\mathrm{Ht}=$ heart, $\mathrm{Sml}=$ small intestine, Kid = kidney, Liv = liver, Lg = lung, SkM = skeletal muscle, St $=$ stomach, $\mathrm{Spl}=$ spleen, $\mathrm{Ov}=$ ovary, Tes = testis). (B) FIG4 is expressed at multiple mouse ages in brain and skeletal muscle. Ages of mice tested were 1, 3, 6, 9 and 20 months. Left lanes are from brain, and right lanes are from quadriceps. The top blot was probed with anti-FIG4, and the bottom blot (loading control) was probed with glyceraldehyde 3-phosphate dehydrogenase (GAPDH). (C) Western blot of protein extracts from $\mathrm{C} 2 \mathrm{C} 12$ cells at various stages of differentiation. Differentiation was induced by serum withdrawal. Cells were differentiated until long myotubes were obviously present (day $8=\mathrm{D} 8$ ). (D) Analysis of wild-type littermate and pale tremor (p/t) mouse skeletal muscle reveals that FIG4 is absent from plt muscle. 
different mouse ages or different developmental stages in vitro; thus we conclude that FIG4 is a ubiquitously expressed component of skeletal muscle. Of note, we verified that FIG4 expression was absent in Fig4-null (that is, plt) skeletal muscle as compared to WT littermates (Figure 1D).

\section{Fig4-null myofibers are reduced in size and exhibit ultrastructural abnormalities}

We began our analysis by examining both light and electron microscopic features of skeletal muscle from Fig4null mice. We examined muscle at 5 weeks of age, since these mice do not survive beyond 6 weeks of age [32]. We also studied a limited number of animals with expression of a Fig4 cDNA transgene under control of the neuron-specific enolase (NSE) promoter [15]. These animals were examined at 4, 8 and 20 months of age.

Routine histopathological analysis of quadriceps, gastrocnemius and diaphragm using H\&E and SDH stains did not reveal any obvious abnormalities in plt skeletal muscle compared to the WT (Figures 2A through 2D). However, the CSA of plt myofibers was significantly smaller than that of their WT counterparts. Quantitation of cross-sectional fiber area revealed that plt myofibers were 55.3\% smaller than WT fibers $(n=400$ total fibers counted from quadriceps in three mice per condition) (Figure 2E). This reduction in size corresponds with the observation that overall quadriceps weight in plt animals was similarly significantly reduced compared to that of age-matched WT littermates (Additional file 1: Figure S1). Of note, overall body weight (which is largely dictated by muscle weight) at 6 weeks of age was also significantly reduced in plt mice (Additional file 1: Figure S2).

Ultrastructural analyses were performed on quadriceps muscle from age-matched WT and plt mice using transmission electron microscopy ( $n=4$ per condition). Overall, muscle ultrastructure in plt mice resembled that of controls (Figures 3A and 3B), though swollen and enlarged mitochondria were occasionally observed in muscle from plt mice ( $\mathrm{M}$ in Figure 3C). We did not, however, detect consistent abnormalities in the triad, the location of the EC coupling machinery, in any plt mouse examined (arrow in Figure 3B).

\section{Increased apoptosis in skeletal muscle and primary myocytes from Fig4-deficient mice}

The combination of reduced muscle fiber size and ultrastructurally abnormal mitochondria led us to examine whether there was increased cell death in plt muscle. TUNEL staining of muscle sections revealed few positive cells in the WT tissue but several positive cells in the mutant tissue (Figure 4A). To better evaluate this finding, we studied isolated myocytes from neonatal WT and plt mice. As with primary cells from other

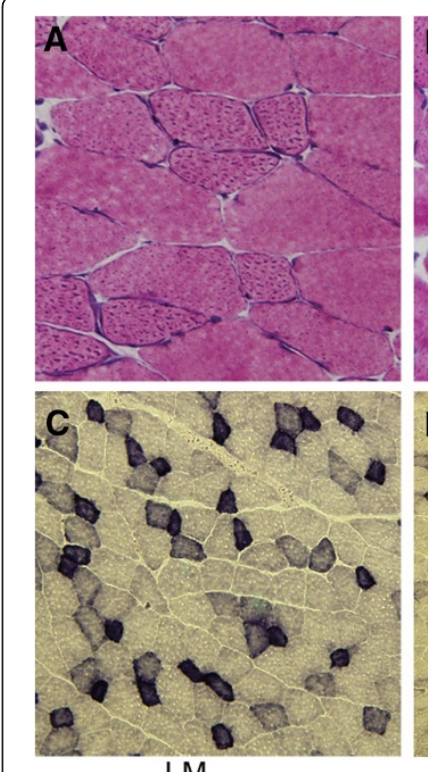

$\mathbf{E}$ Cross Sectional Area $(\mu \mathrm{m} 2)$

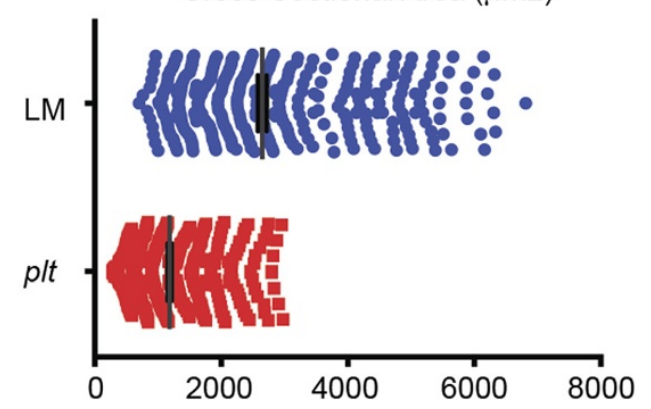

Figure 2 Histopathological analysis of pale tremor $(p / t)$ skeletal muscle. Hematoxylin and eosin staining (A) and (B) and succinate dehydrogenase staining (C) and (D) of cryosections from quadriceps of control (wild-type (WT)/littermate (LM)) and plt animals. Scale bars $=50 \mu \mathrm{m}$. (E) Quantitation of myofiber size from quadriceps muscle of 4-week-old plt and WT animals. There were significant reductions in myofiber size: 2,649 $\mu \mathrm{m}^{2} \pm 70$ for WT vs $1,185 \mu \mathrm{m}^{2} \pm 32$ for $p / t(n=400$ per condition, $P<0.0001)$.

organ systems of the plt mice, cells derived from plt muscle demonstrated abundant vacuolization (Additional file 1: Figure S3). We measured cell death in these primary cell cultures using a TUNEL assay. We observed a significant increase in TUNEL-positive cells in Fig4mutant myocytes, consistent with decreased cell survival (Figure 4B). Of note, despite the vacuolization and impaired survival, Fig4-null myocytes were able to successfully differentiate into myotubes upon serum withdrawal (data not shown). There was also no change in the ability of plt myocytes to proliferate, as determined by bromodeoxyuridine (BrdU) labeling of cells (64\% of cells BrdU-positive in control (116 of 182 cells counted) vs $64 \%$ of cells positive in plt (151 of 235 cells counted). 

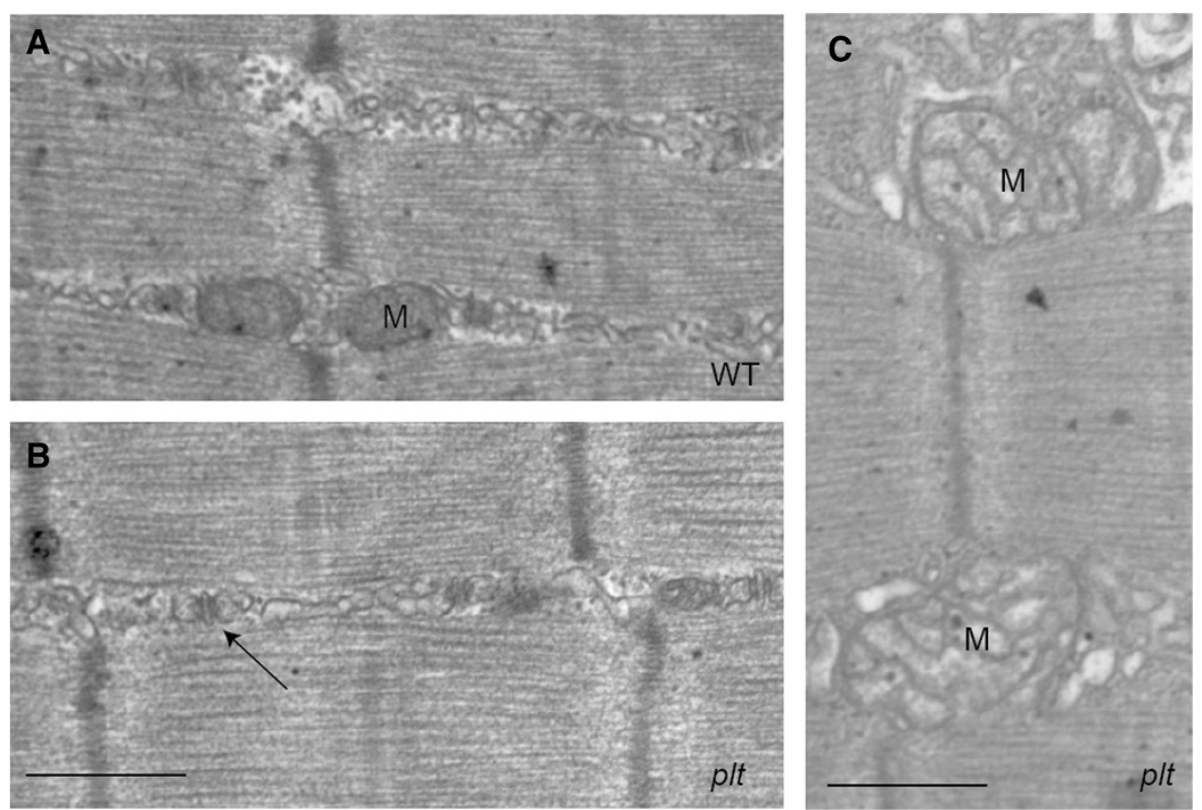

Figure 3 Ultrastructural analysis of pale tremor $(p / t)$ skeletal muscle. Representative photomicrographs from transmission electron microscopic analysis of wild-type/littermate (WT) quadriceps (A) and plt quadriceps (B) and (C). Muscle ultrastructure was generally normal in plt muscle (B) with evidence of normal triads (arrow). There were infrequently observed areas of abnormalities that particularly included swollen and/or dilated mitochondria (M in (C)). Scale bars $=500 \mathrm{~nm}$.

\section{FIG4-mutant skeletal muscle has impaired force generation}

To determine the potential functional effects of Fig4 deficiency in skeletal muscle, we measured force generation from intact muscle fibers. Testing both EDL and soleus muscles revealed statistically significant reductions in specific force generation (12\% to $24 \%$ decrease when adjusted for CSA), indicating mild muscle weakness and impaired force generation in the plt animals (Figure 5).

\section{FIG4-mutant myocytes have normal intracellular calcium dynamics}

Given the previous association between $\mathrm{PI}(3,5) \mathrm{P}_{2}$ and EC coupling and the fact that FIG4 is a known regulator of $\mathrm{PI}(3,5) \mathrm{P}_{2}$ levels, we interrogated intracellular calcium dynamics. We used whole-cell voltage patch-clamping of isolated neonatal mouse myocytes to simultaneously measure voltage-gated L-type calcium currents and intracellular calcium transients. We did not detect abnormalities in either orthograde or retrograde coupling or in L-type
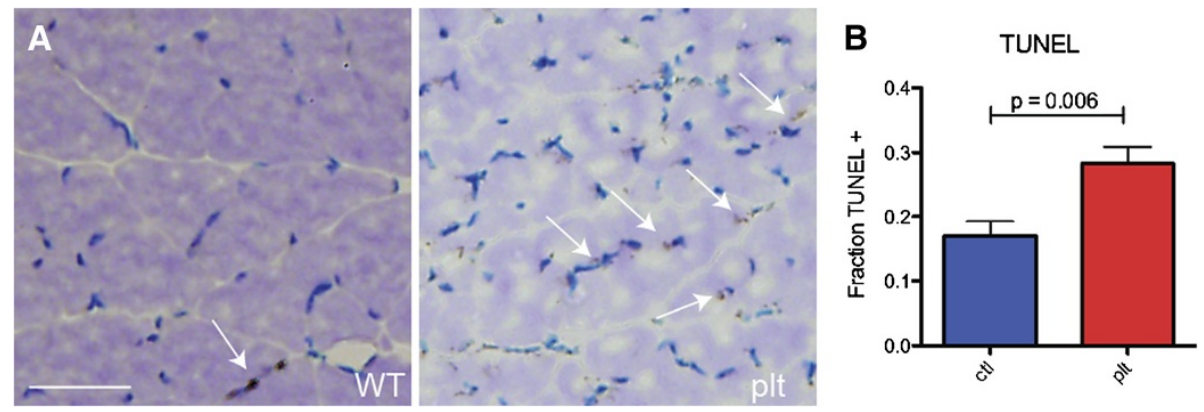

Figure 4 Increased apoptosis in plt skeletal muscle and skeletal myocytes. (A) Terminal deoxynucleotidyl transferase-mediated dUTP nick end labeling (TUNEL) staining was performed on sections from wild-type (WT) and plt skeletal muscle. Arrows point to TUNEL-positive nuclei. There was a qualitative increase in TUNEL-positive fibers in plt muscle (experiment repeated with sections from three different mice per condition). (B) TUNEL staining was performed on myocytes. There was a significant increase in TUNEL-positive cells in plt myocytes vs WT myocytes. Data reflect fraction of 4',6-diamidino-2-phenylindole-positive nuclei that were also TUNEL-positive. Numbers were $0.28 \pm 0.06$ for plt and $0.17 \pm 0.05$ for WT $(P=0.006)$. One hundred cells per experiment were counted, and each experiment was repeated five times (once for each culture derived with cells isolated from five different mice per condition). 

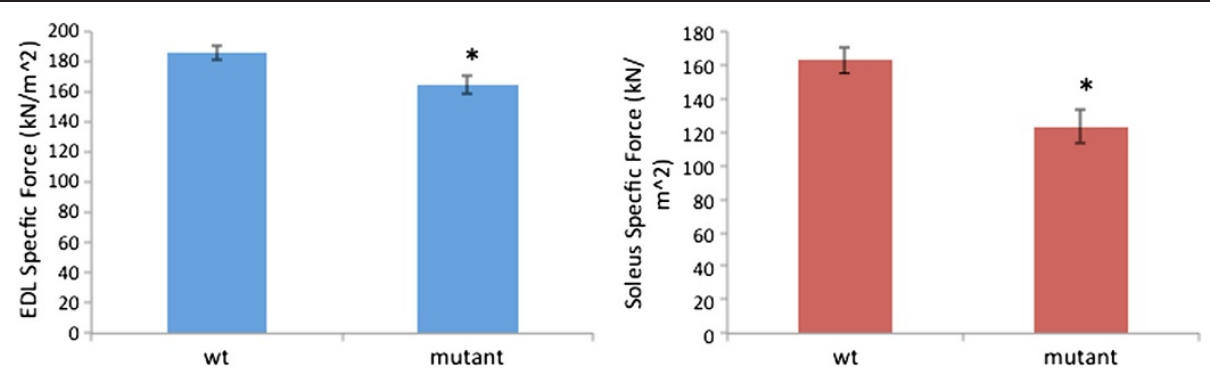

Figure 5 Diminished force production in pale tremor (plt) skeletal muscle. Specific force (normalized to muscle and body weight) was measured for wild-type/littermate (WT) and plt (mutant) skeletal muscle (left panel = extensor digitorum longus, or EDL) (right panel = soleus). Normalized force was significantly reduced in plt muscles ( $n=7$ animals tested per condition). ${ }^{*} P=0.043$ for EDL and $P<0.001$ for soleus.

calcium currents in plt myocytes (Figure 6 and Table 1). Taken in conjunction with the normal histological appearance of the triad, these data suggest that loss of Fig4 is not associated with impaired bidirectional dihydropyridine receptor (DHPR)-RyR1 coupling.

\section{Neuronal expression of Fig4 in plt mice reduces skeletal muscle pathology}

We previously reported that expression of FIG4 under the NSE promoter in plt animals prevents the development of typical phenotypic abnormalities [15]. In fact, the appearance of the transgenic $(\mathrm{Tg})$ animals is indistinguishable from their WT littermates. We examined skeletal muscle in Fig4 $4^{-/-} / \mathrm{TgNSE}$ mice at 4, 8 and 20 months of age. There was no obvious difference in histological appearance between WT and Fig4 $4^{-/} /$TgNSE mice, which is similar to what we observed in the Fig4-null mice. Unlike the null mice, however, Fig4 $4^{-/-} / \mathrm{TgNSE}$ mice exhibited only a very slight alteration in myofiber size $(11 \%$ smaller than WT; $n=5$ animals examined per genotype, $P=0.02$ ) (Figures 7A and 7B). This reduction was statistically significant and occurred despite the fact that overall weight was the same between WT and Fig4 ${ }^{-/-} /$TgNSE animals. $\mathrm{Fig4}^{-/-} / \mathrm{TgNSE}$ muscle did not display any of the ultrastructural changes seen in the Fig4 ${ }^{-1-}$ mice (Figure 7C) and did not exhibit evidence of increased apoptosis.

Last, to determine whether muscle function was altered in Fig $4^{-1-} /$ TgNSE mice, we measured maximum isometric force in soleus and EDL muscles of 18-month-old animals. There was a small (approximately 10\%) but statistically insignificant $(P>0.1)$ decline in force generation between Fig $^{-/-}$/TgNSE mice and their WT littermates (Additional file 1: Figure S4). Taking all our data together, we conclude that expression of Fig4 in neurons is sufficient to correct the development of significant muscle atrophy and programmed cell death in plt skeletal muscle, but not to
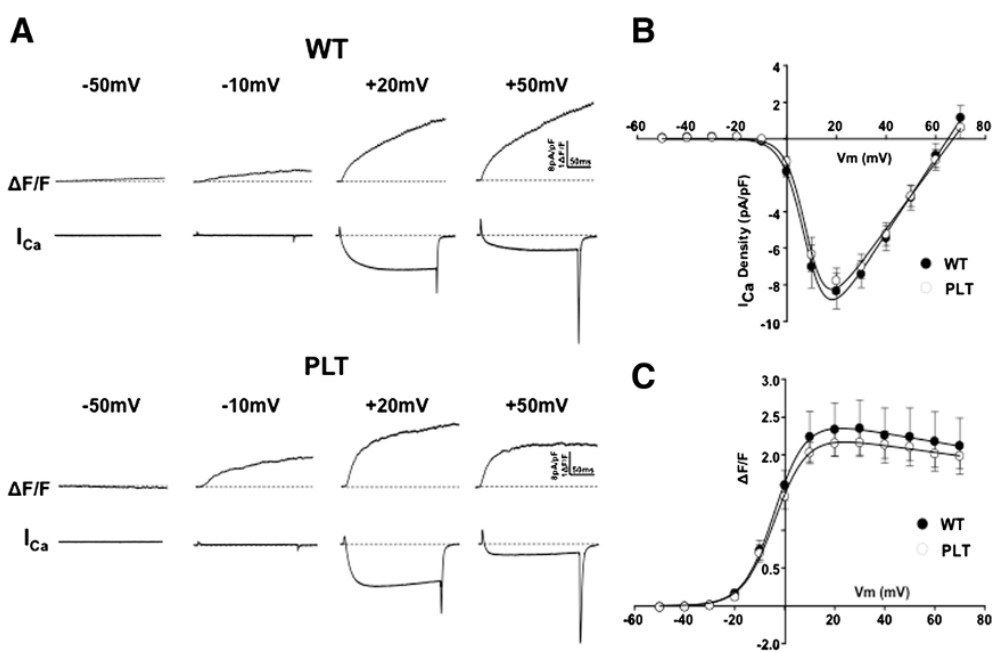

C

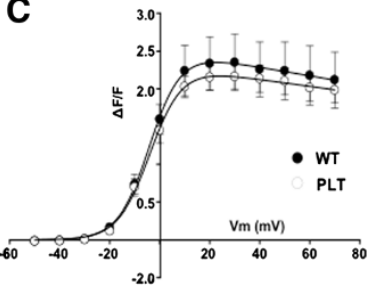

Figure 6 L-currents and voltage-gated $\mathrm{Ca}^{2+}$ release are unaltered in pale tremor (plt) myotubes. (A) Representative whole-cell L-type $\mathrm{Ca}^{2+}$ currents $\left(I_{C_{a}}\right)$ and intracellular $\mathrm{Ca}^{2+}$ transients ( $\Delta \mathrm{F} / \mathrm{F}$ ) obtained following 200-ms depolarizations to the indicated membrane potentials in myotubes derived from either wild-type (WT) mice (upper panel) or plt mice (lower panel). (B) and (C) Average ( \pm SEM) voltage dependence of peak L-type $\mathrm{Ca}^{2+}$ current density (B) and intracellular $\mathrm{Ca}^{2+}$ transients (C) for myotubes derived from either WT mice (filled circles) or plt mice (open circles). 
Table 1 Parameters of fitted current-voltage and fluorescence-voltage $(\Delta F / F-V)$ curves $^{a}$

\begin{tabular}{lccccccc}
\hline & $\boldsymbol{G}_{\max }(\mathbf{n S} / \mathbf{n F})$ & $\boldsymbol{V}_{\mathbf{G} \mathbf{1 / 2}}(\mathbf{m V})$ & $\boldsymbol{k}_{\mathbf{G}}(\mathbf{m V})$ & $\boldsymbol{V}_{\mathbf{R E V}}(\mathbf{m V})$ & $(\Delta \mathbf{F} / \mathbf{F})_{\max }$ & $\boldsymbol{V}_{\mathbf{F} 1 / \mathbf{2}}(\mathbf{m V})$ & $\boldsymbol{k}_{\mathbf{F}}(\mathbf{m V})$ \\
\hline$W T(n=11)$ & $219 \pm 14$ & $9.8 \pm 1.4$ & $5.0 \pm 0.5$ & $65.3 \pm 3.0$ & $2.3 \pm 0.4$ & $-7.8 \pm 1.9$ & $4.4 \pm 0.2$ \\
plt $(\mathrm{n}=11)$ & $194 \pm 15$ & $9.4 \pm 1.3$ & $4.0 \pm 0.4$ & $66.7 \pm 2.7$ & $2.1 \pm 0.2$ & $-5.8 \pm 1.7$ & $4.6 \pm 0.3$ \\
\hline
\end{tabular}

${ }^{a} W T$ wild type.

prevent subtle but reproducible changes in myofiber size in vivo. Of note, we verified by both RNA (reverse transcriptase polymerase chain reaction) and Western blot analysis that there was no appreciable Fig4 RNA or protein in muscle of Fig4 $4^{-/} /$TgNSE mice (data not shown and Figure 1D).

\section{Fig4 haploinsufficiency does not improve the Mtm1-knockout mouse phenotype}

There is a complex interplay between the enzymes that regulate phosphoinositides. One potential avenue for treatment of diseases related to PIP dysregulation is manipulation of other enzyme levels. This point has been demonstrated for the neurological abnormalities associated with Mtmr2 mutation [30]. Mice with a recessive mutation in Mtmr2 exhibit peripheral neuropathy, and aspects of this neuropathy are reversed in the setting of Fig4 haploinsufficiency. Since MTM1 and MTMR2 are highly homologous, we evaluated the effect of haploinsufficiency of Fig4 (in $\mathrm{plt}^{+/-}$mice) on the severe muscle phenotype in knockout mice lacking expression of Mtm1. Generation of $\mathrm{Fig4}^{+/-} / \mathrm{Mtm}^{-/ Y}$ male mice revealed no difference from $M t m 1^{-/ Y}$ mice, with comparable impairments of weight gain, motor function and survival and no statistically significant difference in weight or survival (Figures 8A and $8 \mathrm{~B})$.

By histopathological analysis, we demonstrated similar alterations between $M t m 1^{-/ Y}$ mice and $M t m 1^{-/ Y} / F_{g} 4^{+/-}$ mice. We quantitated myofiber size because size is correlated with disease severity in patients with MTM [36]. There was no clear difference in this parameter (Figure 8B, consistent with the observed lack of functional improvement in the Fig4 $4^{+/-} / M t m 1^{-/ Y}$ animals. In all, haploinsufficiency of Fig4 did not appear to alter the Mtm1 phenotype in any meaningful way.

\section{Discussion}

FIG4 exhibits an important role in central and peripheral nervous system development and homeostasis. The consequences of FIG4 deficiency on other organ systems are less well delineated. In this study, we defined the consequences of Fig4-null mutation on skeletal muscle. We found that (1) global inactivation of Fig4 in the plt mouse is associated with myofiber atrophy and/or hypotrophy, increased apoptosis and diminished specific force production; (2) loss of Fig4 does not alter the structure, EC coupling apparatus or bidirectional DHPR-RyR1 coupling; and (3) reexpression of Fig4 in neurons significantly reduces all observed muscle
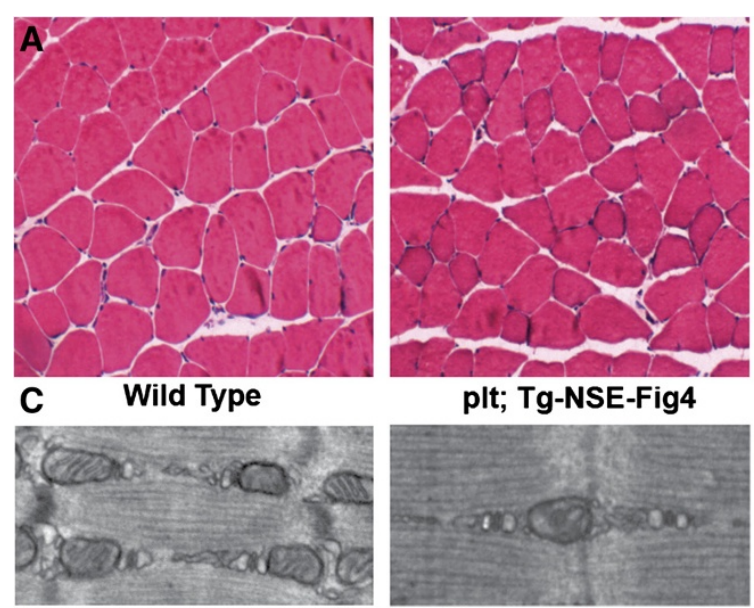

Figure 7 Transgenic (Tg) neuronal rescue of FIG4 expression largely restores plt myofiber size. (A) Hematoxylin and eosin-stained sections of gastrocnemius muscle from wild-type (WT) and Fig4 ${ }^{-1-}$ /NSE-FIG4 (that is, plt with transgenic expression of Fig4 driven by the neuron-specific enolase (NSE) promoter). The plt muscle is essentially indistinguishable from WT, with the exception of mild reduction in fiber size. (B) Quantification of myofiber size from quadriceps muscles of 8-month-old Fig4 ${ }^{-1-}$ /NSE-FIG4 and WT animals. There was a small but significant reduction in total fiber area in transgenically rescued $p / t$ muscle: $2,799 \mu \mathrm{m}^{2} \pm 165$ for WT vs $2,322 \mu \mathrm{m}^{2} \pm 121$ for $p / t(n=60, P=0.02)$. (C) Transmission electron photomicrographs from tibialis anterior muscle. Normal triads and mitochondria were present in both WT and plt/Tg-NSE-Fig4 animals. 

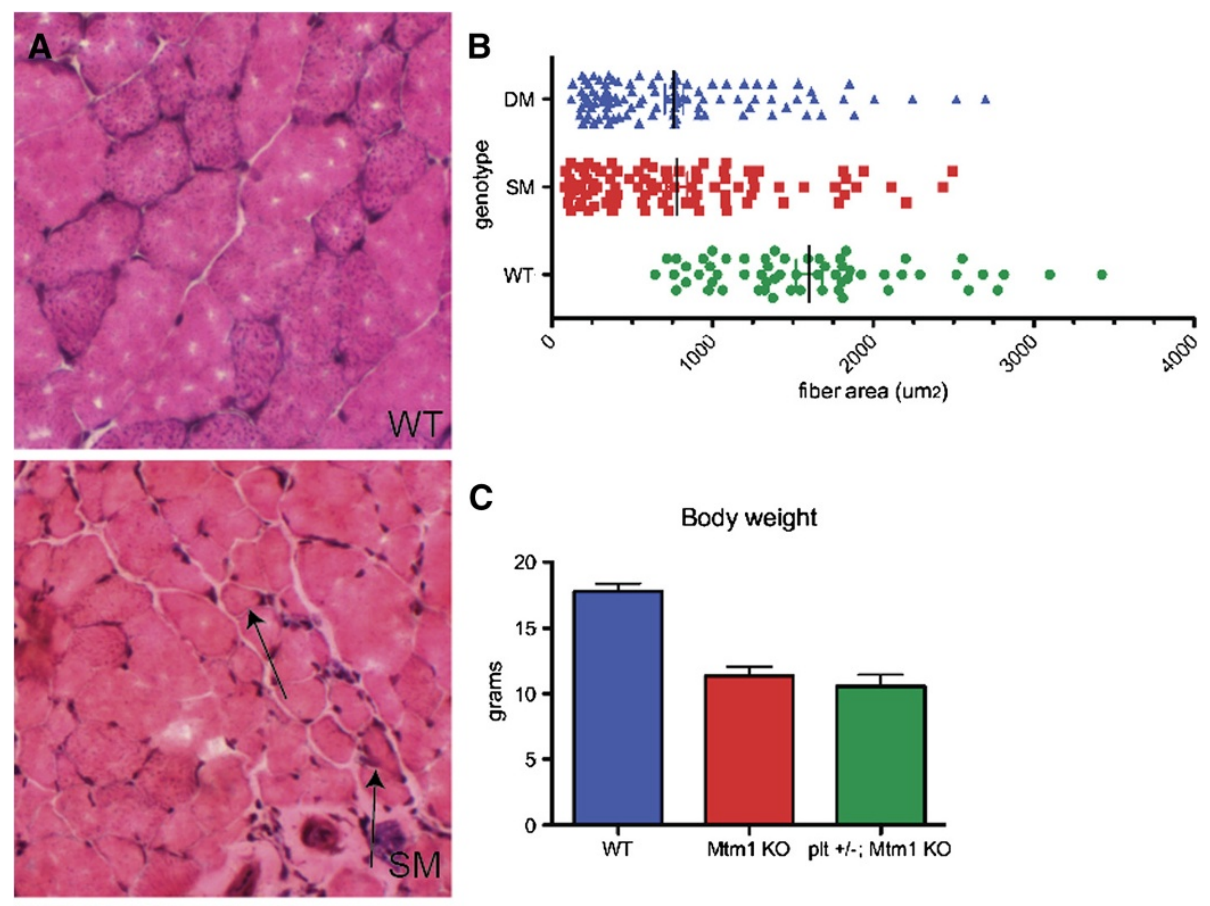

C
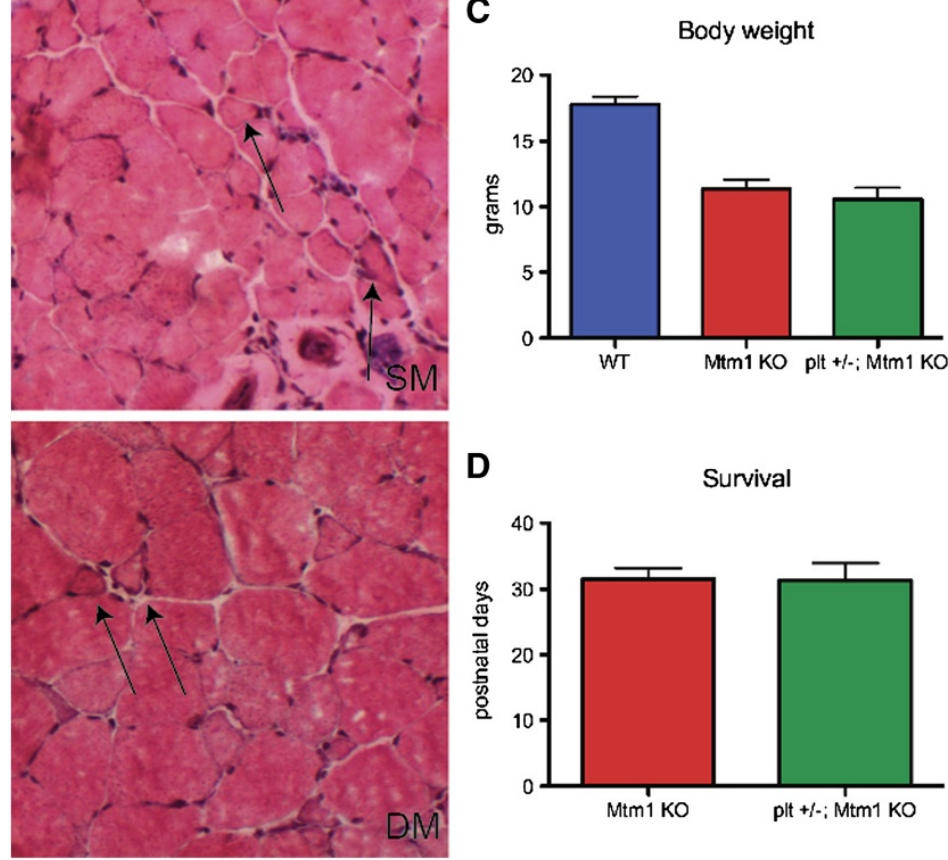

Figure 8 Fig4 haploinsufficiency does not improve the Mtm1-knockout phenotype. (A) Hematoxylin and eosin stain of quadriceps muscle from wild-type (WT; plt ${ }^{+/-}$) (top), Mtm1-knockout (Mtm1-KO) (SM, middle) and plt $t^{+/-} / \mathrm{Mtm} 1-\mathrm{KO}$ (DM, bottom) animals. There was no obvious difference in the appearance of the muscle or in the number of abnormal fibers (arrows). (B) Distribution of fiber area. Fiber area was measured from WT; $\left.p / t^{+/-}\right)$, single-mutant (SM; Mtm1-KO) and double-mutant (DM; $\left.p l t^{+/-} / \mathrm{Mtm} 1-\mathrm{KO}\right)$ skeletal muscles. Thirty fibers from each animal were measured ( $n=4$ for SM and DM, $n=2$ for WT). (C) Terminal body weight (measured in grams). There was significant reduction in the Mtm 1-KO body weight compared to WT. There was no improvement in body weight in $\mathrm{plt} \mathrm{t}^{+/} / \mathrm{Mtm} 1-\mathrm{KO}$ animals. Average values were as follows: $17.8 \pm 0.6 \mathrm{~g}$ for WT $(n=8), 11.4 \pm 0.7 \mathrm{~g}$ for Mtm1-KO $\left(n=9, P<0.0001\right.$ compared to WT) and $10.5 \pm 0.9 \mathrm{~g}$ for $\mathrm{plt} \mathrm{t}^{+/-} / \mathrm{Mtm} 1-\mathrm{KO}(n=5, P=0.48 \mathrm{compared}$ to Mtm1-KO). (D) Haploinsufficiency of Fig4 did not provide any survival benefit. Average survival was $32 \pm 2$ days for Mtm1-KO $(n=9)$ and $31 \pm 3$ days for $\mathrm{plt} \mathrm{t}^{+/-} / \mathrm{Mtm1} 1-\mathrm{KO}(n=5)$.

abnormalities. The implications of these findings are discussed below.

Perhaps the most striking aspect of this study is the fact that Fig4 mutation did not result in a more deleterious direct effect on muscle development and function. In neurons and glia, loss of FIG4 results in severe structural and functional consequences. Given the previously recognized importance of phosphoinositide regulation in skeletal muscle [26,27], we predicted that plt mice would exhibit pronounced changes in muscle structure and function. However, effects of Fig4 ablation on muscle were minimal and largely rescued following neuron-specific restoration of FIG4 expression (Fig4 $4^{-/} /$TgNSE mice). Muscle alterations found to persist in Fig $4^{-/-} / \mathrm{TgNSE}$ mice (for example, $11 \%$ reduction in fiber size) could be explained by either a minor role for FIG4 expression in muscle fiber size determination or a small amount of residual neurogenic atrophy. It is of interest to note the dichotomy between the relatively normal appearance of adult plt muscle in situ and the extensive vacuolarization of plt myocytes in vitro. Similar effects of culturing have been observed in other cell types, including fibroblasts [12] and osteoblasts 
(unpublished manuscript, Lenk GM and Meisler MH). The explanation for this difference is unclear and requires further experimentation.

There are several potential explanations for the lack of a more severe phenotype in skeletal muscle of Fig4-null mice. First of all, the partial reduction of $\mathrm{PI}(3,5) \mathrm{P}_{2}$ likely caused by loss of Fig4 may not be enough to result in significant consequences for muscle development and function. Alternatively, a different PIKfyve protein complex may compensate for the loss of FIG4 to provide sufficient levels of $\mathrm{PI}(3,5) \mathrm{P}_{2}$ to maintain myofiber homeostasis. A third possibility is that $\mathrm{PI}(3,5) \mathrm{P}_{2}$ may be generated in muscle by a different or complementary pathway. For example, a three-position kinase may generate $\mathrm{PI}(3,5) \mathrm{P}_{2}$ from PI(5)P. One current barrier to attempting to distinguish these possibilities is the lack of suitable approaches to measure $\mathrm{PI}(3,5) \mathrm{P}_{2}$ in whole tissues such as skeletal muscle. Development of techniques to aid in measuring $\mathrm{PI}(3,5) \mathrm{P}_{2}$ in situ are required to answer these questions more definitively.

Regardless of the explanation, the fact that there is little, if any, muscle-cell autonomous phenotype in Fig4null animals indicates that FIG4 mutations are unlikely to result in primary muscle disease. However, significant secondary neurogenically mediated myopathic features, such as those observed in plt mice, including reduced muscle fiber CSA and specific force generation, suggest that skeletal muscle changes may contribute to disease pathogenesis. In other words, the myopathic changes described herein may influence disease severity in patients with Charcot-Marie-Tooth disease type 4J and other disorders caused by FIG4 gene mutation.

The lack of impairment in the structure of the EC coupling apparatus or of bidirectional triad coupling in Fig4-null mice was unexpected. Data from the MTMR14knockout mice support the hypothesis that increased levels of $\mathrm{PI}(3,5) \mathrm{P}_{2}$ impair calcium release from the ryanodine receptor (the core component of the EC apparatus), though the underlying mechanism is not clear. Our data imply that reduced levels of $\mathrm{PI}(3,5) \mathrm{P}_{2}$ do not acutely impair voltagegated triad calcium release. Furthermore, the chronic loss of FIG4 from muscle (with the potential implication of chronically reduced $\mathrm{PI}(3,5) \mathrm{P}_{2}$ levels) does not alter the ultrastructural appearance of the triad (that is, the location of the EC coupling machinery). Thus, a requirement for normal levels of $\mathrm{PI}(3,5) \mathrm{P}_{2}$ for EC coupling seems unlikely, though the present data do not completely exclude this possibility. In addition to more direct interrogation of EC coupling in FIG4-deficient mice, another potential future direction to address this issue would be to assess the impact on EC coupling of muscle-specific knockout of PIKfyve, the kinase required for $\mathrm{PI}(3,5) \mathrm{P}_{2}$ generation. Again, however, this would necessitate confirmation of a specific reduction in $\mathrm{PI}(3,5) \mathrm{P}_{2}$ levels in skeletal muscle.
The final significant observation in this study is that reduced Fig4 expression via plt haploinsufficiency does not significantly alter the phenotype of Mtm1-knockout mice. Of note, the MTMR2 gene encodes a protein that is highly homologous to MTM1 [8]. In addition, we previously demonstrated that zebrafish $m t m r 2$ functionally compensates (at least in part) for loss of $m t m 1$, suggesting that MTMR2 and MTM1 are functionally quite similar [37]. However, in contrast to our findings in Mtm1-null mice, Bolino and colleagues found that plt haploinsufficiency rescued neuropathy in Mtmr2-knockout animals [30]. The reasons why reduction of FIG4 levels improved the MTMR2-related neuropathology, but not the muscle pathology, seen in Mtm1-null mice are not clear. This distinction may provide another indication of the nonessential role of FIG4 in skeletal muscle or may reflect different quantitative requirements for PI $(3,5) \mathrm{P}_{2}$ in neurons and muscle or specific differences between mammalian MTMR2 and MTM1.

\section{Conclusions}

We present data demonstrating that FIG4 is required for muscle function but is dispensable for muscle development. In addition, most abnormalities associated with Fig4 mutation appears to be secondary to the severe neuropathy documented in plt mice. Our results do not support a role for FIG4 in EC coupling. Future experiments are needed to more firmly establish the relationship between $\mathrm{PI}(3,5) \mathrm{P}_{2}$ and EC coupling.

\section{Additional file}

Additional file 1: Figure S1. Reduced muscle mass in plt animals. Figure S2. Reduced body mass in plt animals. Figure S3. Vacuoles are present in plt myocytes. Figure S4. Muscle force is restored to normal in Fig4 -/-; Tg;NSE mice.

\section{Abbreviations}

DHPR: Dihydropyridine receptor; EC coupling: Excitation-contraction coupling; NSE: Neuron-specific enolase; PI(3)P: Phosphatidylinositol 3-phosphate; PI(3,5) $\mathrm{P}_{2}$ : Phosphatidylinositol 3,5-bisphosphate;

PIP: Phosphatidylinositol phosphate; plt: Pale tremor mouse; RyR1: Skeletal muscle ryanodine receptor 1; TUNEL: Terminal deoxynucleotidyl transferase-mediated dUTP nick end labeling.

\section{Competing interests}

The authors declare that they have no competing interests.

\section{Authors' contributions}

AR performed the majority of experiments, aided with data interpretation and helped generate the manuscript. XL, DW and MB helped perform the experiments. GL helped with mouse husbandry and manuscript generation. SVB performed and interpreted muscle force experimentation. LG and RTD designed, performed, analyzed and interpreted the myotube voltage-clamp experiments. MM aided with experimental design, data interpretation and manuscript generation. JJD conceived the project, helped with all data interpretation and generated the manuscript. All authors read and approved the final manuscript. 


\section{Acknowledgments}

This work was funded primarily by a grant from the Muscular Dystrophy Association to JJD (MDA186999). This work also was supported in part by National Institutes of Health grants GM24872 (to MHM), AR059646 (to RTD) and 1K08AR054835 (to JJD). GML is a fellow of the Postdoctoral Translational Scholars Program of the Michigan Clinical \& Translational Science Awards (UL1 TR000433). We also acknowledge the support of the Taubman Medical Institute and the Department of Pediatrics at the University of Michigan.

\section{Author details}

'Department of Pediatrics, University of Michigan Medical Center, Ann Arbor, Ml 48109-2200, USA. ${ }^{2}$ Department of Human Genetics, University of Michigan Medical Center, Ann Arbor, Ml 48109-2200, USA. ${ }^{3}$ Department of Pharmacology and Physiology, University of Rochester Medical Center, Rochester, NY 14642, USA. ${ }^{4}$ Molecular and Integrative Physiology, University of Michigan Medical Center, Ann Arbor, Ml 48109-2200, USA. ${ }^{5}$ Neuroscience Graduate Program, University of Michigan, Ann Arbor, MI 48109-2200, USA.

Received: 26 March 2013 Accepted: 29 July 2013

Published: 1 September 2013

\section{References}

1. Di Paolo G, De Camilli P: Phosphoinositides in cell regulation and membrane dynamics. Nature 2006, 443:651-657.

2. De Camilli P, Emr SD, McPherson PS, Novick P: Phosphoinositides as regulators in membrane traffic. Science 1996, 271:1533-1539.

3. Ho CY, Alghamdi TA, Botelho RJ: Phosphatidylinositol-3,5-bisphosphate: no longer the poor PIP. Traffic 2012, 13:1-8.

4. Zolov SN, Bridges D, Zhang Y, Lee WW, Riehle E, Verma R, Lenk GM, Converso-Baran K, Weide T, Albin RL, Saltiel AR, Meisler MH, Russell MW, Weisman LS: In vivo, Pikfyve generates $\mathrm{PI}(3,5) \mathrm{P}_{2}$, which serves as both a signaling lipid and the major precursor for PI5P. Proc Natl Acad Sci USA 2012, 109:17472-17477.

5. Tosch V, Rohde HM, Tronchère H, Zanoteli E, Monroy N, Kretz C, Dondaine N, Payrastre B, Mandel JL, Laporte J: A novel Ptdlns3P and Ptdlns $(3,5) P_{2}$ phosphatase with an inactivating variant in centronuclear myopathy. Hum Mol Genet 2006, 15:3098-3106.

6. Hakim S, Bertucci MC, Conduit SE, Vuong DL, Mitchell CA: Inositol polyphosphate phosphatases in human disease. Curr Top Microbiol Immunol 2012, 362:247-314.

7. Dyson JM, Fedele CG, Davies EM, Becanovic J, Mitchell CA: Phosphoinositide phosphatases: just as important as the kinases. Subcell Biochem 2012. 58:215-279

8. Robinson FL, Dixon JE: Myotubularin phosphatases: policing 3-phosphoinositides. Trends Cell Biol 2006, 16:403-412.

9. Jin N, Chow CY, Liu L, Zolov SN, Bronson R, Davisson M, Petersen JL, Zhang Y, Park S, Duex JE, Goldowitz D, Meisler MH, Weisman LS: VAC14 nucleates a protein complex essential for the acute interconversion of $\mathrm{PI} 3 \mathrm{P}$ and $\mathrm{PI}(3,5) \mathrm{P}_{2}$ in yeast and mouse. EMBO J 2008, 27:3221-3234.

10. Duex JE, Tang F, Weisman LS: The Vac14p-Fig4p complex acts independently of Vac7p and couples PI3,5P $\mathrm{P}_{2}$ synthesis and turnover. J Cell Biol 2006, 172:693-704.

11. Gary JD, Sato TK, Stefan CJ, Bonangelino CJ, Weisman LS, Emr SD Regulation of Fab1 phosphatidylinositol 3-phosphate 5-kinase pathway by Vac7 protein and Fig4, a polyphosphoinositide phosphatase family member. Mol Biol Cell 2002, 13:1238-1251.

12. Chow CY, Zhang Y, Dowling JJ, Jin N, Adamska M, Shiga K, Szigeti K, Shy ME, Li J, Zhang X, Lupski JR, Weisman LS, Meisler MH: Mutation of FIG4 causes neurodegeneration in the pale tremor mouse and patients with CMT4J. Nature 2007, 448:68-72.

13. Winters JJ, Ferguson CJ, Lenk GM, Giger-Mateeva VI, Shrager P, Meisler MH, Giger RJ: Congenital CNS hypomyelination in the Fig4 null mouse is rescued by neuronal expression of the $\mathrm{PI}(3,5) \mathrm{P}_{2}$ phosphatase Fig4. J Neurosci 2011, 31:17736-17751.

14. Ferguson CJ, Lenk GM, Meisler MH: Defective autophagy in neurons and astrocytes from mice deficient in $\mathrm{PI}(3,5) \mathrm{P}_{2}$. Hum Mol Genet 2009, 18:4868-4878.

15. Ferguson CJ, Lenk GM, Jones JM, Grant AE, Winters JJ, Dowling JJ, Giger RJ, Meisler MH: Neuronal expression of Fig4 is both necessary and sufficient to prevent spongiform neurodegeneration. Hum Mol Genet 2012, 21:3525-3534
16. Ikonomov OC, Sbrissa D, Fligger J, Delvecchio K, Shisheva A: ArPIKfyve regulates Sac3 protein abundance and turnover: disruption of the mechanism by Sac3 ${ }^{141 T}$ mutation causing Charcot-Marie-Tooth 4J disorder. J Biol Chem 2010, 285:26760-26764.

17. Lenk GM, Ferguson CJ, Chow CY, Jin N, Jones JM, Grant AE, Zolov SN, Winters JJ, Giger RJ, Dowling JJ, Weisman LS, Meisler MH: Pathogenic mechanism of the FIG4 mutation responsible for Charcot-Marie-Tooth disease CMT4J. PLoS Genet 2011, 7:e1002104.

18. Nicholson G, Lenk GM, Reddel SW, Grant AE, Towne CF, Ferguson CJ, Simpson E, Scheuerle A, Yasick M, Hoffman S, Blouin R, Brandt C, Coppola G, Biesecker LG, Batish SD, Meisler MH: Distinctive genetic and clinical features of CMT4J: a severe neuropathy caused by mutations in the PI (3,5) $\mathrm{P}_{2}$ phosphatase FIG4. Brain 2011, 134:1959-1971.

19. Chow CY, Landers JE, Bergren SK, Sapp PC, Grant AE, Jones JM, Everett $L$, Lenk GM, McKenna-Yasek DM, Weisman LS, Figlewicz D, Brown RH, Meisler MH: Deleterious variants of $F I G 4$, a phosphoinositide phosphatase, in patients with ALS. Am J Hum Genet 2009, 84:85-88.

20. Romero-Suarez S, Shen J, Brotto L, Hall T, Mo C, Valdivia HH, Andresen J, Wacker M, Nosek TM, Qu CK, Brotto M: Muscle-specific inositide phosphatase (MIP/MTMR14) is reduced with age and its loss accelerates skeletal muscle aging process by altering calcium homeostasis. Aging (Albany NY) 2010, 2:504-513.

21. Laporte J, Bedez F, Bolino A, Mandel JL: Myotubularins, a large disease-associated family of cooperating catalytically active and inactive phosphoinositides phosphatases. Hum Mol Genet 2003, 12(Spec No 2):R285-R292

22. Gibbs EM, Feldman EL, Dowling Jj: The role of MTMR14 in autophagy and in muscle disease. Autophagy 2010, 6:819-820.

23. Hnia K, Vaccari I, Bolino A, Laporte J: Myotubularin phosphoinositide phosphatases: cellular functions and disease pathophysiology. Trends $\mathrm{Mol}$ Med 2012, 18:317-327.

24. Pierson CR, Dulin-Smith AN, Durban AN, Marshall ML, Marshall JT, Snyder AD, Naiyer N, Gladman JT, Chandler DS, Lawlor MW, Buj-Bello A, Dowling JJ, Beggs AH: Modeling the human MTM1 p.R69C mutation in murine Mtm 1 results in exon 4 skipping and a less severe myotubular myopathy phenotype. Hum Mol Genet 2012, 21:811-825.

25. Amoasii L, Hnia K, Laporte J: Myotubularin phosphoinositide phosphatases in human diseases. Curr Top Microbiol Immunol 2012, 362:209-233.

26. Dowling JJ, Low SE, Busta AS, Feldman EL: Zebrafish MTMR14 is required for excitation-contraction coupling, developmental motor function and the regulation of autophagy. Hum Mol Genet 2010, 19:2668-2681.

27. Al-Qusairi L, Weiss N, Toussaint A, Berbey C, Messaddeq N, Kretz C, Sanoudou D, Beggs AH, Allard B, Mandel JL, Laporte J, Jacquemond V, Buj-Bello A: T-tubule disorganization and defective excitationcontraction coupling in muscle fibers lacking myotubularin lipid phosphatase. Proc Natl Acad Sci USA 2009, 106:18763-18768.

28. Shen J, Yu WM, Brotto M, Scherman JA, Guo C, Stoddard C, Nosek TM, Valdivia HH, Qu CK: Deficiency of MIP/MTMR14 phosphatase induces a muscle disorder by disrupting $\mathrm{Ca}^{2+}$ homeostasis. Nat Cell Biol 2009, 11:769-776

29. Touchberry CD, Bales IK, Stone JK, Rohrberg TJ, Parelkar NK, Nquyen T, Fuentes O, Liu X, Qu CK, Andresen JJ, Valdivia HH, Brotto M, Wacker MJ: Phosphatidylinositol 3,5-bisphosphate $\left(\mathrm{PI}(3,5) \mathrm{P}_{2}\right)$ potentiates cardiac contractility via activation of the ryanodine receptor. J Biol Chem 2010, 285:40312-40321.

30. Vaccari I, Dina G, Tronchère H, Kaufman E, Chicanne G, Cerri F, Wrabetz L, Payrastre B, Quattrini A, Weisman LS, Meisler MH, Bolino A: Genetic interaction between MTMR2 and FIG4 phospholipid phosphatases involved in Charcot-Marie-Tooth neuropathies. PLoS Genet 2011 7:e1002319.

31. Bolino A, Bolis A, Previtali SC, Dina G, Bussini S, Dati G, Amadio S, Del Carro U, Mruk DD, Feltri ML, Cheng CY, Quattrini A, Wrabetz L: Disruption of Mtmr2 produces CMT4B1-like neuropathy with myelin outfolding and impaired spermatogenesis. J Cell Biol 2004, 167:711-721.

32. Lenk GM, Meisler MH: Mouse models with defects in $\mathrm{PI}(3,5) \mathrm{P}_{2}$ and impaired endolysosome function. Methods Enzymol. in press.

33. Brooks SV, Faulkner JA: Contractile properties of skeletal muscles from young, adult and aged mice. J Physiol 1988, 404:71-82.

34. Avila G, O'Brien JJ, Dirksen RT: Excitation-contraction uncoupling by a human central core disease mutation in the ryanodine receptor. Proc Natl Acad Sci USA 2001, 98:4215-4220. 
35. Avila G, Dirksen RT: Functional effects of central core disease mutations in the cytoplasmic region of the skeletal muscle ryanodine receptor. I Gen Physiol 2001, 118:277-290.

36. Pierson CR, Agrawal PB, Blasko J, Beggs AH: Myofiber size correlates with MTM1 mutation type and outcome in X-linked myotubular myopathy. Neuromuscul Disord 2007, 17:562-568.

37. Dowling JJ, Vreede AP, Low SE, Gibbs EM, Kuwada JY, Bonnemann CG, Feldman EL: Loss of myotubularin function results in T-tubule disorganization in zebrafish and human myotubular myopathy. PLoS Genet 2009, 5:e1000372.

doi:10.1186/2044-5040-3-21

Cite this article as: Reifler et al:: Murine Fig4 is dispensable for muscle development but required for muscle function. Skeletal Muscle 2013 3:21.

\section{Submit your next manuscript to BioMed Central and take full advantage of:}

- Convenient online submission

- Thorough peer review

- No space constraints or color figure charges

- Immediate publication on acceptance

- Inclusion in PubMed, CAS, Scopus and Google Scholar

- Research which is freely available for redistribution 